\title{
Comparison of immunohistochemistry, DNA sequencing and allele-specific PCR for the detection of IDH1 mutations in gliomas
}

\author{
DELPHINE LOUSSOUARN ${ }^{1}$, ANNE-GAËLLE LE LOUPP ${ }^{2}$, JEAN-SÉBASTIEN FRENEL ${ }^{3}$, \\ FRANÇOIS LECLAIR $^{1}$, ANDREAS VON DEIMLING ${ }^{4}$, MAUD AUMONT ${ }^{5}$, \\ STÉPHANE MARTIN $^{6}$, MARIO CAMPONE ${ }^{3}$ and MARC G. DENIS ${ }^{2}$
}

\begin{abstract}
Departments of ${ }^{1}$ Pathology, ${ }^{2}$ Biochemistry, Nantes University Hospital, Nantes; ${ }^{3}$ Department of Medical Oncology, Institut de Cancérologie de l'Ouest - Cancer Center René Gauducheau, Saint Herblain, France; ${ }^{4}$ Department of Neuropathology, Institute of Pathology, University Heidelberg, and Clinical Cooperation Unit Neuropathology, DKFZ, Heidelberg, Germany; ${ }^{5}$ Department of Radiotherapy, Institut de Cancérologie de l'Ouest - Cancer Center René Gauducheau, Saint Herblain; ${ }^{6}$ Department of Neurosurgery, Nantes University Hospital, Nantes, France
\end{abstract}

Received December 22, 2011; Accepted February 13, 2012

DOI: $10.3892 /$ ijo.2012.1404

\begin{abstract}
Previous studies have identified mutations of the isocitrate dehydrogenase 1 (IDH1) gene in more than $70 \%$ of World Health Organization (WHO) grade II and III gliomas. The most frequent mutation leads to a specific amino acid change from arginine to histidine at codon 132 (c.395G >A, p.R132H). IDH1 mutated tumors have a better prognosis than IDH1 non-mutated tumors. The aim of our study was to evaluate and compare the methods of mIDH1 R132H immunohistochemistry, allele-specific PCR and DNA sequencing for determination of IDH1 status. We performed a retrospective study of 91 patients with WHO grade II $(n=43)$ and III $(n=48)$ oligodendrogliomas. A fragment of exon 4 spanning the sequence encoding the catalytic domain of IDH1, including codon 132, was amplified and sequenced using standard conditions. Allele-specific amplification was performed using two forward primers with variations in their 3 ' nucleotides such that each was specific for the wild-type or the mutated variant, and one reverse primer. Immunohistochemistry was performed with mouse monoclonal mIDH1 R132H. DNA was extracted from FFPE sections following macrodissection. IDH1 mutations were found in $55 / 90$ patients $(61.1 \%)$ by direct sequencing. $\mathrm{R} 132 \mathrm{H}$ mutations were found in $47 / 55$ patients $(85.4 \%)$. The results of the allele-specific PCR positively correlated with those from DNA sequencing. Other mutations (p.R132C, p.R132S and pR132G) were found by DNA sequencing in 3 ,
\end{abstract}

Correspondence to: Professor Marc G. Denis, Laboratoire de Biochimie, Institut de Biologie, 9 quai Moncousu, 44093 Nantes Cedex, France

E-mail: marc.denis@chu-nantes.fr

Key words: isocitrate dehydrogenase 1, oligodendrogliomas, immunohistochemistry, allele-specific PCR, sequencing
3 and 2 tumors, respectively (8/55 patients, 14.6\%). mIDH1 $\mathrm{R} 132 \mathrm{H}$ immunostaining was found in the 47 patients presenting the R132H mutation (sensitivity $47 / 47,100 \%$ for this mutation). None of the tumors presenting a wild-type IDH1 gene were stained (specificity 35/35, 100\%). Our results demonstrate that immunohistochemistry using the mIDH1 R132H antibody and allele-specific amplification are highly sensitive techniques to detect the most frequent mutation of the IDH1 gene.

\section{Introduction}

A genome-wide analysis has identified somatic mutations at codon 132 in the isocitrate dehydrogenase 1 (IDH1) gene in $12 \%$ of glioblastomas (1). Mutations in IDH1 were most often observed in young patients with secondary glioblastoma that arise from a low-grade glioma and are associated with an increase in overall survival (1). Subsequent studies have identified high frequencies of IDH1 mutations in diffuse World Health Organization (WHO) grade II and III astrocytomas, oligodendrogliomas and oligoastrocytomas (2-5). IDH1 mutations are very rare in primary glioblastomas (1-6).

IDH1 mutations affect the amino acid, arginine, at position 132 of the amino acid sequence on chromosomal arm $2 q 33.3$ $(1-3,5,7)$. The most frequent mutation consists of a substitution of arginine by histidine (R132H) (7). IDH1 encodes isocitrate dehydrogenase 1 , an enzyme that catalyzes the cytosolic oxidative decarboxylation of isocitrate to $\alpha$-ketoglutarate (8). IDH1 mutations inactivate the normal catalytic activity of IDH1, resulting in a decrease in the levels of $\alpha$-ketoglutarate (8). IDH1 mutations have also gained the function of catalyzing the reduction of $\alpha$-ketoglutarate to 2-hydroxyglutarate (9-11).

IDH1 mutations have been reported as a major prognostic and diagnostic biomarker in gliomas. Patients with IDH1 mutations in gliomas have a better prognosis than patients with gliomas without IDH1 mutations with respect to both overall survival $(1,5,12-15)$ and progression-free survival $(13,16)$. 
A number of methods have been developed to detect IDH1 mutations including DNA sequencing $(4,17)$, PCR-restriction fragment length polymorphism (RFLP) $(18,19)$, PCR-single strand conformation polymorphism (SSCP) (20), pyrosequencing (21,22), and high-resolution melting curve analysis (HRM) $(23,24)$. All of these methods have limitations in terms of sensitivity, ease of use and throughput. Furthermore, most of them are time- and labor-consuming. Recently, mutationspecific antibodies raised against the most frequent mutation $\mathrm{R} 132 \mathrm{H}$, have been developed (25-27). Immunohistochemistry is quick, does not require specific equipment for DNA extraction and analysis, and is always possible, even in the case of small biopsies. The aim of our study was to evaluate and compare the methods of mIDH1 R132H immunohistochemistry, allelespecific PCR and DNA sequencing.

In this study, we show that both allele-specific PCR and mIDH1 immunohistochemistry are highly sensitive and specific techniques that can be used in routine practice to detect the IDH1 R132H mutations.

\section{Materials and methods}

Patients. We performed a retrospective study of 91 patients with a diagnosis of WHO grade II ( $n=43 ; 25$ males and 18 females; mean age, 40.6 years) and WHO grade III ( $n=48 ; 32$ males and 16 females; mean age, 50 years) oligodendrogliomas. The study protocol was approved by the ethics committee and the health authorities according to local requirements. The patients were identified through the database of the Department of Pathology (Nantes University Hospital). The patients in our study underwent brain biopsy or tumor resection between 2000 and 2010 at the Department of Surgery (Nantes University Hospital). For each case, pathological slides were systematically reviewed independently by two neuropathologists in order to assess the diagnosis according to the current WHO classification criteria (28).

Immunohistochemistry detection of IDH1 R132H protein. Immunohistochemistry analysis was performed on a Ventana BenchMark XT immunostainer (Ventana Medical Systems, Inc., Tucson, AZ, USA). Briefly, antigen retrieval was performed with CC2 reagent (Ventana Medical Systems) for $30 \mathrm{~min}$. After washing, tissue sections were incubated with mouse monoclonal mIDH1 R132H hybridoma supernatant at $37^{\circ} \mathrm{C}$ for $32 \mathrm{~min}$. Antibody incubation was followed by incubation with UltraView HRP-conjugated multimer system (Ventana Medical Systems). Antigen detection was performed using UltraView diaminobenzidine chromogen step (Ventana Medical Systems). Slides were then removed from the immunostainer, washed under running water and mounted. Tissues were counterstained with hematoxylin.

IDH1 immunostaining was scored independently by two neuropathologists. Staining was interpreted as positive when tumor cells showed a strong cytoplasmic staining for mIDH1 $\mathrm{R} 132 \mathrm{H}$.

DNA sequencing. DNA was extracted from $10 \mu \mathrm{m}$ FFPE sections following macrodissection using an iPrep system (Invitrogen, Life Technologies SAS, Villebon sur Yvette, France). The $10-\mu \mathrm{m}$ sections corresponded to HES-representative slides selected by the pathologist. A fragment of exon 4 (122 bp) spanning the sequence encoding the catalytic domain of IDH1, including codon 132, was amplified with specific primers (4) and sequenced using standard conditions (Beckman Coulter Genomics, Takeley, UK).

Allele-specific amplification. We designed two forward primers with variations in their 3' nucleotides such that each was specific for the wild-type (R132; GGTAAAACCTATCA TCATAGGTCG) or the mutated variant $(132 \mathrm{H}$; GGGTAAAA CCTATCATCATAGGTCA), and one reverse primer (AS; CAC ATACAAGTTGGAAATTTCTGG). The sequence-specific forward and reverse primers were then combined in 'Primer mix R' (primers R132 and AS), and 'Primer mix H' (primers $132 \mathrm{H}$ and $\mathrm{AS}$ ).

The amplification conditions were optimized for the Rotor Gene 3000 instrument (Qiagen, Courtaboeuf, France). PCR amplifications were performed using the LC480 SYBR-Green mix (Roche Diagnostics, Meylan, France). The reaction mixture contained $10 \mu \mathrm{l}$ of the supplied $2 \mathrm{X}$ master mix, $0.5 \mu \mathrm{l}$ of each primer $(10 \mu \mathrm{M}$ each), and $9 \mu \mathrm{l}$ of the template (50 ng genomic DNA). The cycling conditions were as follows: denaturation for $10 \mathrm{~min}$ at $95^{\circ} \mathrm{C}$, amplification for 45 cycles, with denaturation for $10 \mathrm{sec}$ at $95^{\circ} \mathrm{C}$, annealing for $15 \mathrm{sec}$ at $63^{\circ} \mathrm{C}$ and extension for $20 \mathrm{sec}$ at $72^{\circ} \mathrm{C}$. The specific 117-bp (for R132) or 118-bp (for $132 \mathrm{H}) \mathrm{PCR}$ products were amplified, and the cycle threshold $(\mathrm{Ct})$ value were determined for a fixed normalized fluorescence of 0.05 .

\section{Results}

Clinical data. Our study population was composed of 91 patients with a diagnosis of WHO grade II oligodendrogliomas ( $\mathrm{n}=43 ; 25$ males and 18 females; mean age, 40.6 years) and WHO grade III ( $n=48 ; 32$ males and 16 females; mean age, 50 years) oligodendrogliomas. The tumor locations were frontal in 60 cases (66\%), temporal in 19 cases $(20.8 \%)$, parietal in six cases $(6.6 \%)$. There were six thalamic tumors $(6.6 \%)$. The patients underwent biopsy in 33 cases (36.2\%) and surgical resection in 58 cases $(63.8 \%)$.

DNA sequencing. DNA was amplified in 90 cases (98.9\%). IDH1 mutations were found in $55 / 90$ patients $(61.1 \%)$ by direct sequencing. Among these patients, the R132H mutation was found in $47 / 55$ patients $(85.4 \%)$. Other mutations (p.R132C, p.R132S and pR132G) were found by DNA sequencing in 3, 3 and 2 tumors, respectively (8/55 patients, $14.6 \%)$.

Allele specific PCR. We first determined the sensitivity of our assay, and compared it to DNA sequencing. We selected a tumor that was found to present the $\mathrm{R} 132 \mathrm{H}$ mutation, and another one which presented the wild-type mutation. We performed serial dilutions of the mutated DNA in a background of wild-type DNA. These samples were then amplified and analyzed by DNA sequencing. The results are presented in Fig. 1. Using this assay, we detected the $\mathrm{R} 132 \mathrm{H}$ mutation down to the $25 \%$ dilution, which is in agreement with previous reports (24).

The same samples were analyzed by allele-specific amplification. For each sample, the $\mathrm{Ct}$ value was determined for the R132 (control) PCR, and for the 132H (mutation-specific) $\mathrm{PCR}$, and the difference was calculated ( $\Delta \mathrm{Ct}$, Fig. $2 \mathrm{~A})$. 

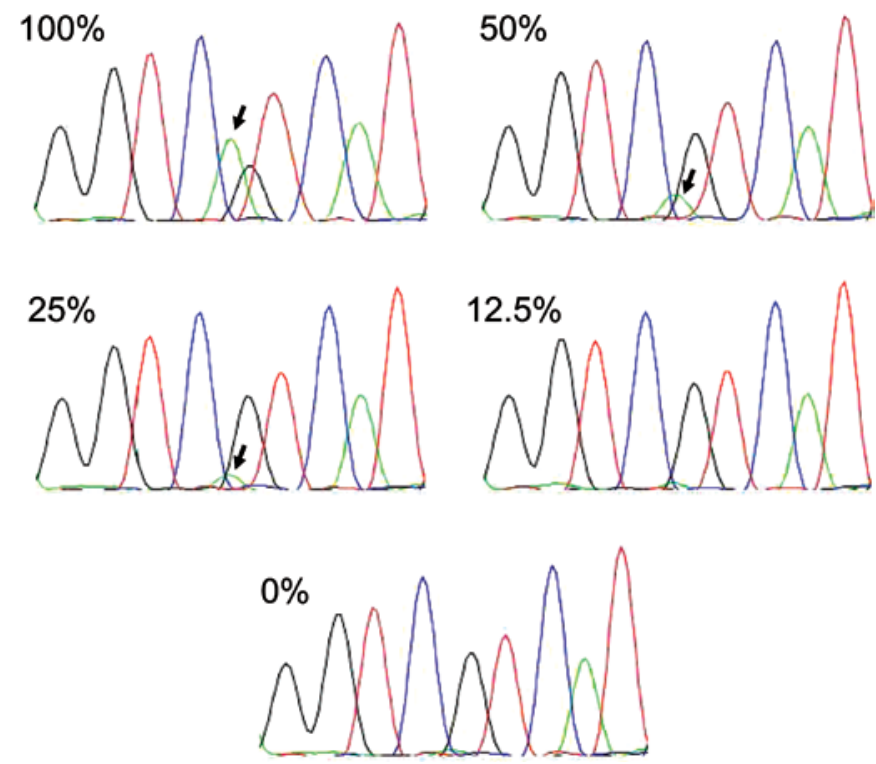

Figure 1. Sensitivity of DNA sequencing for the detection of the R132H mutation. Serial dilutions of the IDH1 R132H mutant $(100,50,25$ and $12.5 \%)$ in a background of wild-type DNA $(0 \%)$ were performed and analyzed by DNA sequencing. The arrow shows the mutation site.
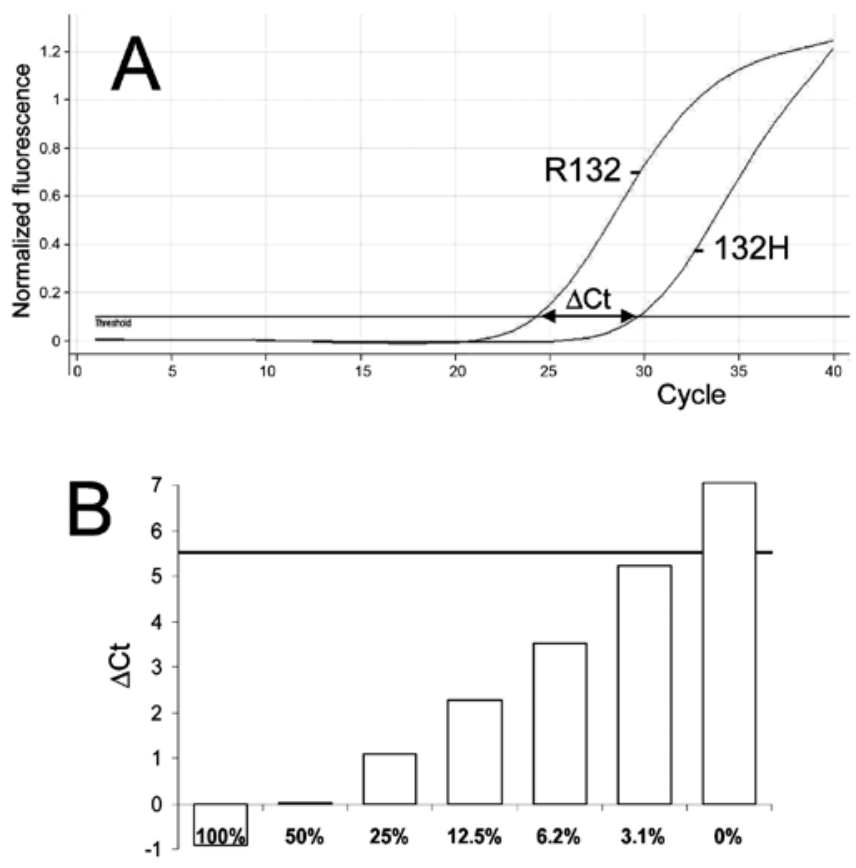

Dilution of IDH1 R132H DNA in normal DNA

Figure 2. Sensitivity of the allele-specific PCR assay. The same samples used for Fig. 1 were analyzed by allele-specific amplification. (A) For each sample, the cycle threshold $(\mathrm{Ct}$ ) value was determined for the R132 (control) PCR, and for the $132 \mathrm{H}$ (mutation specific) PCR, and the difference was calculated $(\Delta \mathrm{Ct}$ ). (B) Results obtained with the serial dilutions. The lane indicates the cut-off value of 5.5.

The lower the amount of mutated DNA in the sample, the higher the $\Delta \mathrm{Ct}$ value. The wild-type DNA yielded a $\Delta \mathrm{Ct}$ value of $\sim 7$ (Fig. 2B). The last dilution, containing 3.1\% DNA from the mutated tumor (and 96.9\% DNA from the wild-type tumor) gave a $\Delta \mathrm{Ct}$ value of $\sim 5$, and was thus determined as
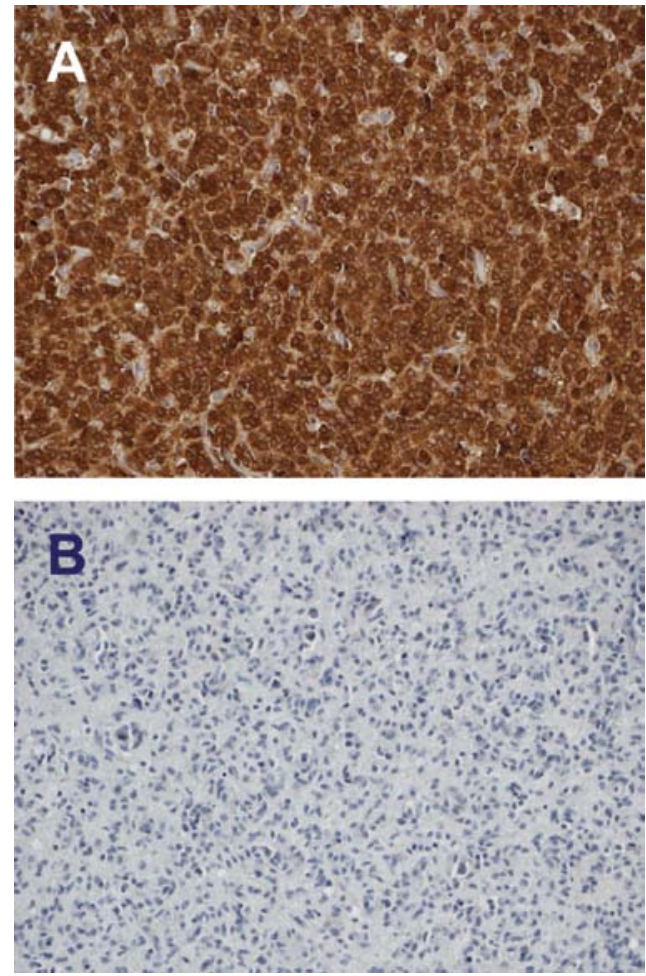

Figure 3. mIDH1 R132H immunostaining of oligodendroglial tumor cells. (A) Tumor presenting a $\mathrm{R} 132 \mathrm{H}$ mutation by direct sequencing and allele-specific PCR (x200). (B) Tumor presenting an IDH1 R132C mutation (x200).

positive using a cut-off value of 5.5 (Fig. 2B). Therefore, we concluded that our allele-specific amplification was 8-10-fold more sensitive than DNA sequencing performed under standard conditions.

Using this assay, we then tested the 90 tumors of our series which could be amplified. The results of the allele-specific PCR positively correlated with DNA sequencing: tumors harboring the $\mathrm{R} 132 \mathrm{H}$ mutation were also found to be positive with this assay, whereas wild-type tumors and tumors presenting other IDH1 mutations yielded negative results.

IDH1 immunohistochemistry. mIDH1 R132H immunostaining was found in 47 patients (47/91). Immunostaining was found in the 47 tumors presenting the R132H mutation (sensitivity $47 / 47$, $100 \%$ for this mutation). None of the tumors presenting the wild-type IDH1 gene were stained (specificity 35/35, 100\%). The positive cases showed a strong cytoplasmic diffuse staining of neoplastic cells (Fig. 3A). This cytoplasmic immunostaining was sometimes associated with a weak nuclear staining. No staining of endothelial cells or of perivascular lymphocytes was observed. No staining was found in the tumors presenting the wild-type IDH1 gene (data not shown) or the other mutations R132C, R132S and R132G (Fig. 3B).

\section{Discussion}

IDH1 mutations have been reported as a powerful diagnostic and prognostic biomarker in gliomas $(5,12,13,25,29-31)$. Recent publications have demonstrated that IDH1 status determination is of major diagnostic interest for the diagnosis of infiltrative glioma versus reactive gliosis, particularly in the case of small 
biopsies $(25,29)$. Furthermore, IDH1 mutations aid in the discrimination of oligodendrogliomas and oligoastrocytomas from other clear cell 'oligodendrocyte-like' tumors (30). A number of studies have demonstrated a favorable prognostic impact of IDH1 mutations on overall survival and progressionfree survival of patients with gliomas $(1,5,12,13,15)$. As many of these studies are retrospective, it is not clear whether the IDH1 status is a predictive marker for outcome to treatment, or a prognostic marker. Van den Bent et al (16) conducted a prospective randomized study in WHO grade III anaplastic oligodendrogliomas. They demonstrated that IDH1 mutations were of major prognostic significance in grade III oligodendrogliomas, but not predictive of treatment response.

A number of techniques have been used to detect IDH1 mutations. DNA sequencing is often considered to be the 'gold standard', although it is perhaps the less sensitive approach. Other molecular biology techniques often require specific equipment (e.g. pyrosequencing and HRM). Furthermore, PCR tubes must be opened following amplification, to add reagent(s) or transfer product to a new tube. These steps are time consuming, and constitute a major risk of contamination. In contrast, the allele-specific PCR-based technique does not require further manipulation of PCR products. We have previously shown that allele-specific PCR is a very robust technique to detect point mutation with high sensitivity and throughput $(32,33)$. We therefore decided to compare the methods of mIDH1 R132H immunohistochemistry, allele-specific PCR and DNA sequencing in our series of oligodendrogliomas.

Using direct sequencing, we found IDH1 mutations in $55 / 90(61.1 \%)$ patients with WHO grade II and III oligodendrogliomas. IDH1 mutations were present in 32/43 (74.4\%) WHO grade II tumors and 23/48 (47.9\%) WHO grade III tumors. Our results are comparable with the results of other studies that found IDH1 mutations in $71-82 \%$ of OII, $67-86 \%$ of O III, $78-100 \%$ of OA II and $66-100 \%$ of OA III tumors $(2,4,5,7)$.

We demonstrated in our study that $\mathrm{R} 132 \mathrm{H}$ mutations were found in $47 / 55$ patients $(85.4 \%)$. The other mutations with direct sequencing were observed in 8/55 patients (14.6\%). Hartman et al, in a large series of 1010 grade II and grade III gliomas demonstrated that $93 \%$ of IDH1 mutations consisted of a substitution of the amino acid, arginine, by histidine $(\mathrm{R} 132 \mathrm{H})$ (7). In our study, R132H mutations were found in $85.4 \%$ of the patients. The other mutations were represented by p.R132C, p.R132S and pR132G in 3, 3 and 2 patients, respectively $(8 / 55$ patients $(14.6 \%)$. The correlation between the IDH1 mutation status and clinical outcome of these patients is being investigated.

The results from allele specific PCR positively correlated with the results from direct sequencing. We were not able to show a higher mutation rate with allele-specific PCR, possibly due to the fact that our samples were enriched by macrodissection, and contained a sufficient percentage of cancers cells to allow standard sequencing to detect these mutations. We have prospectively analyzed every new glioma diagnosed at our centre since this study was completed. Recently, we found three cases that were positive by immunohistochemistry and allelespecific PCR, but negative by direct sequencing (unpublished data).

In our study, we used the mutation-specific IDH1 antibody for the most frequent mutation $(\mathrm{R} 132 \mathrm{H})$ first described by
Capper et al (26). Using this antibody, mIDH1 R132H immunostaining was found in the 47 patients presenting the $\mathrm{R} 132 \mathrm{H}$ mutation (sensitivity $47 / 47,100 \%$ for this mutation). None of the tumors presenting the wild-type IDH1 gene were stained (specificity 35/35, 100\%). No immunostaining was found in R132C-, R132S- and R132G-mutated tumors. Our results are comparable to those of Capper et al (25). The global sensitivity of $\mathrm{R} 132 \mathrm{H}$ immunohistochemistry in this series was $94 \%$ due to the absence of detection of the other mutations by immunohistochemistry, but the sensitivity for R132H mutations was $100 \%$. The specificity of R132H immunohistochemistry was $100 \%$. In our samples the proportion of neoplastic cells as assessed by the pathologist was always above $10 \%$, even for WHO grade II tumors. The proportion of neoplastic cells may be problematic for direct sequencing in the cases of small stereotactic biopsies for low-grade glioma. In the study of Capper et al (25), direct sequencing was negative in eight cases $(8 / 186)$ that were found positive by immunohistochemistry. After resequencing the tumors, these cases were found to present an IDH1 mutation.

In conclusion, we designed a sensitive assay to detect the R132H mutation of the IDH1 gene. Many samples can be analyzed simultaneously in $\sim 1 \mathrm{~h}$ without any subsequent manipulation of the PCR products. Furthermore, this assay is based on the use of the fluorescent dye SYBR-Green I but relies on gene-specific fluorescent probes and is therefore much more cost-effective. Immunohistochemistry is also very useful as it is quick, does not require specific equipment, and allows the identification of a few R132H IDH1-mutated tumor cells in small biopsies. Both techniques can be used to rapidly screen a large number of tumor samples for the most frequent IDH1 mutations.

\section{Acknowledgements}

We thank Nadege Friquet for her technical assistance.

\section{References}

1. Parsons DW, Jones S, Zhang X, et al: An integrated genomic analysis of human glioblastoma multiforme. Science 321: 1807- 1812, 2008.

2. Balss J, Meyer J, Mueller W, Korshunov A, Hartmann C and von Deimling A: Analysis of the IDH1 codon 132 mutation in brain tumors. Acta Neuropathol 116: 597-602, 2008.

3. Ichimura K, Pearson DM, Kocialkowski S, et al: IDH1 mutations are present in the majority of common adult gliomas but rare in primary glioblastomas. Neuro Oncol 11: 341-347, 2009.

4. Watanabe T, Nobusawa S, Kleihues P and Ohgaki H: IDH1 mutations are early events in the development of astrocytomas and oligodendrogliomas. Am J Pathol 174: 1149-1153, 2009.

5. Yan H, Parsons DW, Jin G, et al: IDH1 and IDH2 mutations in gliomas. N Engl J Med 360: 765-773, 2009.

6. Bleeker FE, Lamba S, Leenstra S, et al: IDH1 mutations at residue p.R132 (IDH1(R132)) occur frequently in high-grade gliomas but not in other solid tumors. Hum Mutat 30: 7-11, 2009.

7. Hartmann C, Meyer J, Balss J, et al: Type and frequency of IDH1 and IDH2 mutations are related to astrocytic and oligodendroglial differentiation and age: a study of 1,010 diffuse gliomas. Acta Neuropathol 118: 469-474, 2009.

8. Zhao S, Lin Y, Xu W, et al: Glioma-derived mutations in IDH1 dominantly inhibit IDH1 catalytic activity and induce HIF-1alpha. Science 324: 261-265, 2009.

9. Dang L, White DW, Gross S, et al: Cancer-associated IDH1 mutations produce 2-hydroxyglutarate. Nature 462: 739-744, 2009.

10. Reitman ZJ, Jin G, Karoly ED, et al: Profiling the effects of isocitrate dehydrogenase 1 and 2 mutations on the cellular metabolome. Proc Natl Acad Sci USA 108: 3270-3275, 2011. 
11. Xu W, Yang H, Liu Y, et al: Oncometabolite 2-hydroxyglutarate is a competitive inhibitor of alpha-ketoglutarate-dependent dioxygenases. Cancer Cell 19: 17-30, 2011.

12. Nobusawa S, Watanabe T, Kleihues P and Ohgaki H: IDH1 mutations as molecular signature and predictive factor of secondary glioblastomas. Clin Cancer Res 15: 6002-6007, 2009.

13. Sanson M, Marie Y, Paris S, et al: Isocitrate dehydrogenase 1 codon 132 mutation is an important prognostic biomarker in gliomas. J Clin Oncol 27: 4150-4154, 2009.

14. van den Bent MJ, Carpentier AF, Brandes AA, et al: Adjuvant procarbazine, lomustine, and vincristine improves progressionfree survival but not overall survival in newly diagnosed anaplastic oligodendrogliomas and oligoastrocytomas: a randomized European Organisation for Research and Treatment of Cancer phase III trial. J Clin Oncol 24: 2715-2722, 2006.

15. Wick W, Hartmann C, Engel C, et al: NOA-04 randomized phase III trial of sequential radiochemotherapy of anaplastic glioma with procarbazine, lomustine, and vincristine or temozolomide. J Clin Oncol 27: 5874-5880, 2009.

16. van den Bent MJ, Dubbink HJ, Marie Y, et al: IDH1 and IDH2 mutations are prognostic but not predictive for outcome in anaplastic oligodendroglial tumors: a report of the European Organization for Research and Treatment of Cancer Brain Tumor Group. Clin Cancer Res 16: 1597-1604, 2010.

17. Seiz M, Tuettenberg J, Meyer J, et al: Detection of IDH1 mutations in gliomatosis cerebri, but only in tumors with additional solid component: evidence for molecular subtypes. Acta Neuropathol 120: 261-267, 2010.

18. Bujko M, Kober P, Matyja E, et al: Prognostic value of IDH1 mutations identified with PCR-RFLP assay in glioblastoma patients. Mol Diagn Ther 14: 163-169, 2010.

19. Meyer J, Pusch S, Balss J, et al: PCR- and restriction endonuclease-based detection of IDH1 mutations. Brain Pathol 20: 298-300, 2010

20. Kang MR, Kim MS, Oh JE, et al: Mutational analysis of IDH1 codon 132 in glioblastomas and other common cancers. Int J Cancer 125: 353-355, 2009.

21. Felsberg J, Wolter M, Seul H, et al: Rapid and sensitive assessment of the IDH1 and IDH2 mutation status in cerebral gliomas based on DNA pyrosequencing. Acta Neuropathol 119: 501-507, 2010.
22. Setty P, Hammes J, Rothamel T, et al: A pyrosequencing-based assay for the rapid detection of IDH1 mutations in clinical samples. J Mol Diagn 12: 750-756, 2010.

23. Boisselier B, Marie Y, Labussiere M, et al: COLD PCR HRM: a highly sensitive detection method for IDH1 mutations. Hum Mutat 31: 1360-1365, 2010.

24. Lin J, Qian J, Yao DM, et al: Rapid and reliable detection of IDH1 R132 mutations in acute myeloid leukemia using high-resolution melting curve analysis. Clin Biochem 44: 779-783, 2011.

25. Capper D, Weissert S, Balss J, et al: Characterization of $\mathrm{R} 132 \mathrm{H}$ mutation-specific IDH1 antibody binding in brain tumors. Brain Pathol 20: 245-254, 2009.

26. Capper D, Zentgraf H, Balss J, Hartmann C and von Deimling A: Monoclonal antibody specific for IDH1 R132H mutation. Acta Neuropathol 118: 599-601, 2009.

27. Kato Y, Jin G, Kuan CT, McLendon RE, Yan H and Bigner DD: A monoclonal antibody IMab-1 specifically recognizes IDH1R132H, the most common glioma-derived mutation. Biochem Biophys Res Commun 390: 547-551, 2009.

28. Kleihues P and Sobin LH: World Health Organization classification of tumors. Cancer 88: 2887, 2000.

29. Camelo-Piragua S, Jansen M, Ganguly A, Kim JC, Louis DN and Nutt CL: Mutant IDH1-specific immunohistochemistry distinguishes diffuse astrocytoma from astrocytosis. Acta Neuropathol 119: 509-511, 2010.

30. Capper D, Reuss D, Schittenhelm J, et al: Mutation-specific IDH1 antibody differentiates oligodendrogliomas and oligoastrocytomas from other brain tumors with oligodendroglioma-like morphology. Acta Neuropathol 121: 241-252, 2011

31. Weller M, Felsberg J, Hartmann C, et al: Molecular predictors of progression-free and overall survival in patients with newly diagnosed glioblastoma: a prospective translational study of the German Glioma Network. J Clin Oncol 27: 5743-5750, 2009.

32. Bouancheau D, Buecher B, Jarry A, et al: The PPAR(gamma) K422Q mutation does not contribute to troglitazone inefficiency in colon cancer treatment. Cancer Lett 224: 111-116, 2005.

33. Jarry A, Masson D, Cassagnau E, Parois S, Laboisse C and Denis MG: Real-time allele-specific amplification for sensitive detection of the BRAF mutation V600E. Mol Cell Probes 18: 349-352, 2004 